\title{
Analogue simulation of gravitational waves in a 3+1-dimensional Bose-Einstein condensate
}

\author{
Daniel Hartley, ${ }^{1, *}$ Tupac Bravo, ${ }^{1}$ Dennis Rätzel, ${ }^{1}$ Richard Howl, ${ }^{1}$ and Ivette Fuentes ${ }^{1,2}$ \\ ${ }^{1}$ Faculty of Physics, University of Vienna, Boltzmanngasse 5, 1090 Wien, Austria \\ ${ }^{2}$ School of Mathematical Sciences, University of Nottingham, \\ University Park, Nottingham NG7 2RD, United Kingdom
}

(Received 27 December 2017; published 17 July 2018)

\begin{abstract}
The recent detections of gravitational waves (GWs) by the LIGO and Virgo collaborations have opened the field of GW astronomy, intensifying interest in GWs and other possible detectors sensitive in different frequency ranges. Although strong GW producing events are rare and currently unpredictable, GWs can in principle be simulated in analogue systems at will in the lab. Simulation of GWs in a manifestly quantum system would allow for the study of the interaction of quantum phenomena with GWs. Such predicted interaction is exploited in a recently proposed Bose-Einstein condensate (BEC) based GW detector. In this paper, we show how to manipulate a BEC to mimic the effect of a passing GW. By simultaneously varying the external potential applied to the BEC, and an external magnetic field near a Feshbach resonance, we show that the resulting change in speed of sound can directly reproduce a $\mathrm{GW}$ metric. We also show how to simulate a metric used in the recently proposed BEC based GW detector, to provide an environment for testing the proposed metrology scheme of the detector. Explicit expressions for simulations of various GW sources are given. This result is also useful to generally test the interaction of quantum phenomena with GWs in a curved spacetime analogue experiment.
\end{abstract}

DOI: 10.1103/PhysRevD.98.025011

\section{INTRODUCTION}

In the 36 years since the seminal proposal of Unruh to measure an acoustic analogue to Hawking radiation from a "sonic horizon" in a fluid [1], interest in analogue simulation of gravitational fields has grown from theoretical proposals to experiments in numerous systems. These include Bose-Einstein condensates (BECs) [2-4], water waves [5,6] and optical fibers [7] among others. Particular interest has been shown in using the phonon field in a BEC, since this is a quantum system and so allows for the study of how nonclassical properties, such as entanglement, are modified or generated by simulated gravitational fields. This has recently culminated in the first observation of the entanglement of acoustic Hawking radiation [8], potentially providing clues to fundamental questions for quantum gravity, such as the information paradox. In addition to Hawking radiation from a waterfall horizon, other proposed simulations using BECs have included conformal Schwarzschild black holes $[9,10]$, rotating black holes [11], Friedmann-Robertson-Walker geometries $[12,13]$, inflation $[14,15]$ and extensions to Einstein's general relativity, such as aether fields [9]. In past work [16], two of us have considered the simulation

*Corresponding author: daniel.hartley@univie.ac.at. of gravitational waves (GWs) in $1+1$ dimensions. These are perturbations of spacetime generated by a changing quadrupole moment of a mass distribution, and have recently been detected in a milestone moment in science [17-21]. This has led to a new field of GW astronomy, enabling the exploration of the Universe through gravitational as well as electromagnetic radiation. Simulating GWs in fluid systems could be of astronomical interest, for example, in studying GWs in numerically challenging, strong-field regimes. Furthermore, since BECs are quantum systems, this could enable the study of predicted effects such as particle creation in GW backgrounds [22], and quantum decoherence due to GWs [23]. While we are interested in simulating the effect of GWs, simulating the evolution of a GW itself on a curved background has also been proposed in [24], where a metamaterial emulates a curved background spacetime and two-photon states model the evolution of a $\mathrm{GW}$ on that background.

Simulating GWs with BECs could also be used in studies of a proposed BEC GW detector [25-27]. This detector consists of a BEC constrained to a rigid trap with a prepared quantum state of phonons, such as a two-mode squeezed state. The transformation induced by the GW produces mode-mixing and phonon creation in a phenomenon resembling the dynamical Casimir effect [28,29], making the final state distinguishable from the probe state, i.e., decreasing the fidelity between the initial and final states. 
The lower the fidelity between the probe and final state, the better the estimation. Nonclassical squeezed states allow for quantum metrology techniques resulting in better estimation than a classical device. At resonance, mode-mixing or phonon creation are maximized, giving rise to optimal parameter estimation. Such a quantum resonance process is absent in laser interferometers since the frequencies of the GWs are far from the optical regime. However, using resonance to detect GWs was the concept behind the first GW detector proposals, Weber bars, which are metal objects measuring meters in length. The $\mathrm{GW}$ resonance in the BEC detector is similar since the much smaller size, $\mathcal{O}(\mu \mathrm{m})$, is compensated for by a much smaller speed of sound, $\mathcal{O}(\mathrm{mm} / \mathrm{s})$ compared to $\mathcal{O}(\mathrm{km} / \mathrm{s})$ [30]. However, the BEC detector can be cooled to considerably lower temperatures, $\mathcal{O}(n K)$, and is a strictly quantum device. This allows for the use of quantum metrology and, therefore, sensitivities that are inaccessible to classical devices [31]. A discussion of the viability of such a detector and further details can be found in [25]. Further studies of the viability of such a detector are in progress and this article is part of this effort. Simulating the effect of a GW derived in [25] could be useful for testing the metrological scheme proposed in $[25,26]$.

Here we extend the work on $1+1$ GWs to the simulation of $3+1 \mathrm{GWs}$ in BECs in a covariant formalism [16]. With this extension, all properties of a GW can be simulated, such as its polarization and propagation vector, and the conformal factor in front of the analogue metric no longer diverges. The paper is outlined as follows. In Sec. II we present the spacetime metric of a GW and the acoustic metric in a BEC. In Sec. III we derive and demonstrate the simulation of a GW metric in $3+1$ dimensions, as well as the metric derived in [25]. Examples of GWs are presented in Sec. IV, giving explicit forms of the flow velocities needed to simulate the effect of commonly investigated GW sources, including compact binary inspirals and neutron star spin down. In Sec. V we reduce the metric derived in Sec. III to $1+1$ dimensions and compare this to previously published work in [16], and we conclude in Sec. VI.

\section{A. Definitions and conventions}

Throughout this paper, we use the metric signature $(-,+,+,+)$; the coordinates used are Minkowski coordinates given by $(c t, x, y, z)$ unless otherwise stated; and the Minkowski metric in these coordinates is given by

$$
\eta_{\mu \nu}=\operatorname{diag}(-1,1,1,1) \text {. }
$$

\section{GRAVITATIONAL WAVES AND THE ACOUSTIC METRIC}

\section{A. GW spacetime metric}

We first consider the general form of the metric tensor perturbed by a GW. For a single source GW far from the source, the metric tensor can be expressed as $[32,33]$

$$
g_{\mu \nu}^{(g w)}=\eta_{\mu \nu}+\epsilon h_{\mu \nu}
$$

where $\eta_{\mu \nu}$ is the flat Minkowski metric defined in Sec. I A, and $h_{\mu \nu}$ is some perturbation corresponding to the passing $\mathrm{GW}$, parametrized by $\epsilon$, where $|\epsilon| \ll 1$. Standard notation omits this $\epsilon$ and applies the condition $\left|h_{\mu \nu}\right| \ll 1$, but we use $\epsilon$ here as a global perturbation scale factor for consistency and clarity.

\section{Transverse traceless gauge}

This perturbation $h_{\mu \nu}$ can be expressed in the transverse traceless (TT) gauge, in coordinates $x_{\mathrm{TT}}^{\mu}$, for a GW traveling in the $\hat{z}$ direction as [33]

$$
h_{\mu \nu}^{\mathrm{TT}}=\left(\begin{array}{cccc}
0 & 0 & 0 & 0 \\
0 & h_{+}(t) & h_{\times}(t) & 0 \\
0 & h_{\times}(t) & -h_{+}(t) & 0 \\
0 & 0 & 0 & 0
\end{array}\right),
$$

where $h_{+}$and $h_{\times}$are time-dependent functions corresponding to two "polarizations" of the GW. These $h_{+}$and $h_{\times}$ functions are typically called "strain" functions. We ignore the $z$ dependence of the strain functions, as the wavelength of a $\mathrm{GW}$ is typically much longer than the width of a BEC (for example, the GWs detected by the LIGO and Virgo collaborations have wavelengths exceeding $10^{6} \mathrm{~m}$ ). Outside the source of the GWs, these strain functions obey the simple wave equation

$$
\eta^{\rho \sigma} \partial_{\rho} \partial_{\sigma} h_{\mu \nu}^{T T}=0
$$

We introduce the GW metric in this gauge, as it is the clearest and most widely known, despite not necessarily being the most physically useful.

\section{Fermi normal coordinates}

Any metric in linearized gravity of the form of Eq. (2) has a "gauge freedom," namely a choice of small coordinate transformation in any arbitrary direction. Consider a linearized coordinate transformation with some function $\zeta$, such that

$$
x^{\mu} \rightarrow x^{\mu}+\epsilon \zeta^{\mu}\left(x^{\mu}\right)
$$

Under such a coordinate transformation, the metric in Eq. (2) transforms as

$\eta_{\mu \nu}+\epsilon h_{\mu \nu} \rightarrow \eta_{\mu \nu}+\epsilon\left[h_{\mu \nu}-\partial_{\nu} \zeta_{\mu}-\partial_{\mu} \zeta_{\nu}\right]+\mathcal{O}\left(\epsilon^{2}\right)$.

Hence, making a coordinate transformation $x_{T T}^{\mu} \rightarrow x^{\mu}=$ $x_{T T}^{\mu}+\epsilon \zeta^{\mu}$ from the TT gauge with the function 


$$
\begin{aligned}
\zeta^{\mu}= & \left(\frac{1}{4 c}\left(2 x y \partial_{t} h_{\times}+\left(x^{2}-y^{2}\right) \partial_{t} h_{+}\right),\right. \\
& \left.\frac{1}{2}\left(x h_{+}+y h_{\times}\right), \frac{1}{2}\left(x h_{\times}-y h_{+}\right), 0\right),
\end{aligned}
$$

the metric perturbation in Eq. (3) in these new coordinates is

$$
h_{\mu \nu}^{\mathrm{TT}} \rightarrow h_{\mu \nu}=\left(\begin{array}{cc}
-h_{00} & \mathbf{0}^{\mathrm{T}} \\
\mathbf{0} & \mathbb{I}_{3}
\end{array}\right)+\mathcal{O}\left(\epsilon^{2}\right)
$$

where $\mathbb{I}_{n}$ is the $n$-dimensional identity matrix, and

$$
h_{00}=-\frac{1}{2 c^{2}}\left(2 x y \partial_{t}^{2} h_{\times}+\left(x^{2}-y^{2}\right) \partial_{t}^{2} h_{+}\right) .
$$

These coordinates are Fermi normal coordinates, and are the inertial frame limit of the "proper detector frame." This metric perturbation can also be derived by considering coordinates matching proper length and time, then linearizing the metric with respect to $R=\sqrt{\eta_{i j} x^{i} x^{j}}$ (derived for example in [33], Sec. I.3.3). One of the most useful features of these coordinates is that they match the laboratory coordinates of an experiment in free fall, e.g., a drag free satellite orbiting the Earth. It is also a good approximation for the suspended mirrors of the LIGO experiments. For notational convenience, we also define

$$
\begin{aligned}
H_{00}(t, x, y) & =\int_{0}^{t} h_{00}\left(t^{\prime}, x, y\right) c d t^{\prime} \\
& =-\frac{1}{c}\left(x y \partial_{t} h_{\times}+\frac{1}{2}\left(x^{2}-y^{2}\right) \partial_{t} h_{+}\right) .
\end{aligned}
$$

\section{B. Acoustic metric}

To simulate a GW metric in a BEC, we will follow the description of a BEC on a general background metric given in $[13,34,35]$. This description models the BEC as a barotropic, irrotational and inviscid fluid, in a covariant formalism. We are interested in (i) simulating a spacetime metric using a quantum system and (ii) simulating the effects of spacetime dynamics on a phononic field. In both cases we require a covariant formalism that enables us to properly describe a general relativistic spacetime and its effects on quantum fields. The formalism developed in $[13,34,35]$ enables us to do so. We point out that the system that we consider here is a regular BEC, as those currently demonstrated in the laboratory. This system is usually described with nonrelativistic quantum mechanics. However, in (ii) we are taking into account the underlying spacetime background on which the BEC sits on, which requires the covariant treatment mentioned above. We point out that we are not considering a system that is moving with relativistic speeds or has excitations with relativistic energies. Such a relativistic system would also need to be described by the same formalism since covariance is also necessary.

Any BEC which can be described as a superfluid is automatically barotropic and inviscid. In the superfluid regime, the BEC is described by a classical mean field $\phi$ expressed as

$$
\phi=\sqrt{\rho} e^{i \theta},
$$

with quantum fluctuations $\hat{\psi}$, defined in terms of the total field $\hat{\Phi}$ as

$$
\hat{\Phi}=\phi(1+\hat{\psi}) .
$$

We are interested in the behavior of these fluctuations in the "phononic" regime. The relativistic phononic regime condition can be written explicitly as [13]

$$
|k| \ll \frac{\sqrt{2}}{\xi}\left(1+\frac{\hbar^{2}}{2 m^{2} \xi^{2} u_{0}^{2}}\right) \min \left[1, \frac{m u_{0} \xi}{\sqrt{2} \hbar}\right],
$$

where $k$ is the spatial frequency of a phononic excitation of the BEC, $u$ is the flow velocity of the BEC defined as

$$
u_{\mu}=\frac{\hbar}{m} \partial_{\mu} \theta
$$

and $\xi$ is the "healing length" defined as

$$
\xi=\frac{1}{\sqrt{\lambda \rho}}
$$

$\lambda$ encodes the strength of the interaction, defined in terms of the interaction potential $U$ as

$$
U\left(\phi^{\dagger} \phi, \lambda\right)=\frac{1}{2} \lambda\left|\phi^{\dagger} \phi\right|^{2}+\cdots
$$

where extra terms are $\phi^{6}$ interactions and higher, which we ignore here. The interaction strength $\lambda$ is related to the scattering length $a$ by

$$
\lambda=8 \pi a .
$$

Taking the nonrelativistic limit of this condition, we find that the phonons should have wavelengths far longer than the healing length $\xi$. In this regime, and with certain additional assumptions about the mean-field properties, the fluctuations obey a relativistic Klein-Gordon-like equation

$$
\frac{1}{\sqrt{-G}} \partial_{\mu}\left(\sqrt{-G} G^{\mu \nu} \partial_{\nu} \hat{\psi}\right)=0
$$

for a tensor $G_{\mu \nu}$ with determinant $G$, called the "acoustic metric." The general acoustic metric is given by (see Appendix B) 


$$
G_{\mu \nu}=\frac{\rho c}{c_{s}}\left(g_{\mu \nu}+r \frac{v_{\mu} v_{\nu}}{c^{2}}\right)
$$

where $g_{\mu \nu}$ is the background spacetime metric, $\rho$ is the bulk density defined as $\rho=\phi^{*} \phi, r$ is related to the speed of sound $c_{s}$ as

$$
r=1-\frac{c_{s}^{2}}{c^{2}}
$$

and $v$ is the normalized flow velocity defined as

$$
v_{\mu}=\frac{c u_{\mu}}{|u|}
$$

The speed of sound $c_{s}$ is defined as

$$
c_{s}^{2}=\frac{c^{2} c_{0}^{2} /|u|^{2}}{1+c_{0}^{2} /|u|^{2}},
$$

where

$$
c_{0}^{2}=\frac{\hbar^{2}}{2 m^{2}} \rho \partial_{\rho}^{2} U(\rho, \lambda)=\frac{\hbar^{2}}{2 m^{2}} \lambda \rho .
$$

The flow velocity $v$ is normalized as

$$
g^{\mu \nu} v_{\mu} v_{\nu}=-c^{2} .
$$

Note that the definition of the flow velocity imposes irrotationality, i.e.,

$$
\partial_{\mu} u_{\nu}=\partial_{\nu} u_{\mu}
$$

Due to the global phase symmetry of the Lagrangian that Eq. (18) is derived from, there is a conserved current. The conservation of this current can be expressed as

$$
\nabla_{\mu}\left(\rho u^{\mu}\right)=0
$$

also called the continuity equation, where $\nabla_{\mu}$ is the covariant derivative with respect to $g_{\mu \nu}$. The velocity normalization $|u|$ and density $\rho$ can also be directly related to the internal and external potentials $U$ and $V$ as

$$
|u|^{2}=c^{2}+\frac{\hbar^{2}}{m^{2}}\left\{V+\frac{\partial U(\rho, \lambda)}{\partial \rho}-\frac{\nabla_{\mu} \nabla^{\mu} \sqrt{\rho}}{\sqrt{\rho}}\right\} .
$$

\section{GRAVITATIONAL WAVE SIMULATION}

In this paper, we present two results corresponding to different types of simulation. The first in Sec. III A is a direct simulation of the GW metric in Eq. (2). The second result in Sec. III B is a simulation of the acoustic metric derived in [25], to test the proposed metrological scheme.

\section{A. GW metric simulation}

The goal of this section is to directly simulate a $\mathrm{GW}$ spacetime, such that the acoustic metric $G_{\mu \nu}$ has the form

$$
G_{\mu \nu}^{(\mathrm{GW})}=\eta_{\mu \nu}+h_{\mu \nu}
$$

We start with the GW metric in Fermi normal coordinates, as introduced in Sec. II A 2. If we consider a BEC at rest in these coordinates, i.e., $v_{\mu}=-c \delta_{\mu}^{0}$, where the background metric is the flat Minkowski metric, then the acoustic metric has the form

$$
G_{\mu \nu}^{(\mathrm{SIM})}=\frac{\rho c}{c_{s}}\left(\begin{array}{cc}
-c_{s}^{2} / c^{2} & \mathbf{0}^{\mathrm{T}} \\
\mathbf{0} & \mathbb{I}_{3}
\end{array}\right) .
$$

It should be noted that the density is not completely unrestricted; the choice of flow velocity restricts the density through the continuity equation. From the definition of the flow velocity in Eq. (14) and the choice of normalized velocity above,

$$
\frac{\hbar}{m} \partial_{\mu} \theta=-|u| c \delta_{\mu}^{0}
$$

which implies that $|u|$ can only be a function of time. Hence, with the continuity equation [Eq. (B16)], for this particular choice of normalized velocity, we must have

$$
\frac{\partial_{t} \rho}{\rho}=-\frac{\partial_{t}|u|}{|u|}
$$

\section{Bulk properties}

Comparison of Eqs. (8) and (29) suggests that, to simulate the GW metric, we must modulate the speed of sound as

$$
c_{s}^{2}=c_{s 0}^{2}\left(1+\epsilon h_{00}\right),
$$

where $c_{s 0}$ is the speed of sound in the absence of a simulated GW. If we rescale the time coordinate as

$$
c_{s 0} \tau=\left(\frac{c_{s 0}^{2}}{c^{2}}\right) c t
$$

then the acoustic metric has the form

$$
G_{\mu^{\prime} \nu^{\prime}}^{(\mathrm{SIM})}=\frac{\rho c}{c_{s}}\left(\begin{array}{cc}
-1-\epsilon h_{00} & \mathbf{0}^{\mathrm{T}} \\
\mathbf{0} & \mathbb{I}_{3}
\end{array}\right)
$$

which matches the desired metric in Eq. (28) up to a conformal factor. The conformal factor will be discussed further later in this subsection, as well as in Sec. III C. 


\section{Implementation}

As mentioned earlier in Sec. III A, we must have $|u|$ dependent on time only. Furthermore, having a density that is changing in time in the absence of flows implies changing the local atom number density in the BEC in a uniform and precisely controlled way, which seems experimentally unfeasible. If it is possible to control the density independently of the flows, then modulating the density to match the speed of sound sets the conformal factor in Eq. (34) to be constant, and thus physically irrelevant. If it is not feasible to control the density without inducing flows, then we must conclude that the density must be constant in time, and thus

$$
\partial_{t}|u|=-\frac{|u| \partial_{t} \rho}{\rho}=0,
$$

so $|u|$ is constant in both space and time. This conclusion can also be drawn from the chemical potential $\mu$ of the BEC. If the BEC is stationary, then $\mu$ is constant. Since

$$
\mu=\frac{i \hbar \partial_{t} \phi}{\phi}=m c|u|,
$$

we can conclude that $|u|$ is constant. Using Eq. (27), we can see that all of these restrictions on the density, speed of sound and flows are only achievable by balancing the external potential $V$ and internal interaction strength $\lambda$ to modulate the speed of sound while leaving the density constant in time. Specifically, we must have

$$
V=-\lambda \rho-\frac{m^{2}}{\hbar^{2}}\left(c^{2}-|u|^{2}\right)+\frac{\nabla^{2} \sqrt{\rho}}{\sqrt{\rho}} .
$$

It is well known that the interaction strength in a BEC can be modulated with an external magnetic field around a Feshbach resonance (see for example [36]). Defining

$$
\lambda=\lambda_{0}+\epsilon \lambda_{1}
$$

and

$$
V=V_{0}+\epsilon V_{1}
$$

for "unperturbed" interaction strength $\lambda_{0}$ and external potential $V_{0}$, we must have

$$
V_{1}=-\lambda_{1} \rho
$$

From the definitions of $c_{s}$ and $c_{0}$ in Eqs. (22) and (23) it is straightforward to show that the interaction strength perturbation must have the form

$$
\lambda_{1}=\frac{\lambda_{0}}{r_{0}} h_{00}
$$

and thus

$$
V_{1}=-\frac{\lambda_{0} \rho}{r_{0}} h_{00},
$$

where

$$
r_{0}=1-\frac{c_{s 0}^{2}}{c^{2}}
$$

The density may still vary over space, with a shape determined by the "unperturbed" external potential $V_{0}$ as usual. Physically, this would result in a BEC cloud "not moving" in time (no flows, fixed density distribution), but with a carefully balanced trapping potential and applied magnetic field changing the speed of sound. This is somewhat analogous to modulating the refractive index in a dielectric, a scheme which has also been explored for its applications in analogue gravity (for example, in [7,37]). It should be noted that implementing the conditions presented in this section does not necessarily result in an exact simulation, as the effective metrics in Eqs. (34) and (28), with the above speed of sound perturbation, differ by a conformal factor, as in the simulation of various black hole geometries in $[10,13,38]$. Explicitly, in our case,

$$
\begin{aligned}
G_{\mu \nu}^{(\mathrm{SIM})} & =\frac{\rho c}{c_{s}} G_{\mu \nu}^{(\mathrm{GW})} \\
& =\frac{\rho c}{c_{s 0}}\left(1-\frac{\epsilon}{2} h_{00}+\mathcal{O}\left(\epsilon^{2}\right)\right) G_{\mu \nu}^{(\mathrm{GW})} .
\end{aligned}
$$

The conformal factor will be discussed further in Sec. III C. While a GW is a coordinate-independent physical effect, the simulation presented here reproduces elements of a metric in a particular coordinate system, and is thus not a coordinate-independent solution.

\section{Nonrelativistic limit}

In the explicitly nonrelativistic limit, the spatial flows are much slower than the speed of light, so $u^{0} \rightarrow c$. The interaction strength must also be weak, so $c_{0} \ll c$. From the definition of $c_{s}$ in Eq. (22), it is clear that we must have $c_{s} \ll c$. In this regime, the phononic regime dispersion relation condition in Eq. (13) reduces to

$$
|k| \ll \frac{\sqrt{2}}{\xi} .
$$

We also assume that the term $\hbar^{2} \partial_{t}^{2} \phi / m c^{2}$ can be neglected; i.e., the excitation energy of each boson is much smaller than its mass energy. The equation governing the evolution of the field $\phi$ becomes the Gross-Pitaevskii equation,

$$
i \hbar \partial_{t} \phi=\left(-\frac{\hbar^{2}}{2 m} \nabla^{2}+V^{\mathrm{NR}}+\lambda^{\mathrm{NR}}|\phi|^{2}\right) \phi
$$

where the external potential and interaction strength are related to those defined in Sec. II B by 


$$
\begin{aligned}
V^{\mathrm{NR}} & =\frac{\hbar^{2}}{2 m} V, \\
\lambda^{\mathrm{NR}} & =\frac{\hbar^{2}}{2 m} \lambda .
\end{aligned}
$$

In the nonrelativistic limit, these are given by

$$
\begin{aligned}
& V_{1}^{\mathrm{NR}}=\epsilon \frac{m c_{s 0}^{2}}{2 c^{2}}\left(2 x y \partial_{t}^{2} h_{\times}+\left(x^{2}-y^{2}\right) \partial_{t}^{2} h_{+}\right), \\
& \lambda_{1}^{\mathrm{NR}}=-\epsilon \frac{m c_{s 0}^{2}}{2 \rho c^{2}}\left(2 x y \partial_{t}^{2} h_{\times}+\left(x^{2}-y^{2}\right) \partial_{t}^{2} h_{+}\right) .
\end{aligned}
$$

\section{B. GW effect simulation}

The goal of this section is to simulate the acoustic metric given in [25], to test the metrological scheme proposed in $[25,26]$. This metric has the form

$$
G_{\mu \nu}^{(g w)}=\frac{\rho_{0} c}{c_{s 0}}\left(\eta_{\mu \nu}+h_{\mu \nu}+r_{0} \frac{v_{0 \mu} v_{0 \nu}}{c^{2}}\right),
$$

where $\rho_{0}, c_{s 0}$ and $v_{0}$ are the properties of the mean field $\phi$ "unperturbed" by a simulated GW. In [25], the BEC is considered to be at rest $\left(v_{0 \mu}=-c \delta_{\mu}^{0}\right)$ in the TT frame, so this is the condition that we will simulate with. Such a simulation can also be done with a BEC at rest in Fermi normal coordinates, which is not the initial condition considered in [25], and this solution is presented in the Appendix A.

\section{Acoustic metric with background $G W$}

The metric perturbation $h$ in Fermi normal coordinates is given in Eq. (8). Going from the TT frame to Fermi normal coordinates, the flow velocity transforms as

$$
\begin{aligned}
v_{T T}^{\mu} & =c \delta_{0}^{\mu} \\
\rightarrow v^{\mu} & =c\left(1-\frac{\epsilon}{2} h_{00},-\frac{\epsilon}{2} \partial_{x} H_{00},-\frac{\epsilon}{2} \partial_{y} H_{00}, 0\right) .
\end{aligned}
$$

Hence, the acoustic metric has the form

$$
G_{\mu \nu}^{(g w)}=\frac{\rho_{0} c}{c_{s 0}}\left(\begin{array}{cccc}
-c_{s 0}^{2} / c^{2}-\epsilon\left(1+r_{0}\right) h_{00} & \frac{r_{0} \epsilon}{2} \partial_{x} H_{00} & \frac{r_{0} \epsilon}{2} \partial_{y} H_{00} & 0 \\
\frac{r_{0} \epsilon}{2} \partial_{x} H_{00} & & & \\
\frac{r_{0} \epsilon}{2} \partial_{y} H_{00} & & \mathbb{I}_{3} & \\
0 & &
\end{array}\right) \text {. }
$$

This is the form of the acoustic metric that we will simulate.

\section{Acoustic metric with simulated GW}

To simulate the metric in Eq. (53), we perturb the bulk properties of the BEC in Fermi normal coordinates (i.e., lab frame). When constructing the simulation, we consider the background metric to be the flat Minkowski metric in Eq. (1), with no GW. Let the density, speed of sound and flow velocity be respectively described as

$$
\begin{gathered}
\rho=\rho_{0}+\epsilon \rho_{1}, \\
c_{s}^{2}=c_{s 0}^{2}+\epsilon c_{s 1}^{2},
\end{gathered}
$$

and

$$
v=\left(v_{0}, \epsilon v_{1}, \epsilon v_{2}, 0\right),
$$

with normalization

$$
|u|=|u|_{0}+\epsilon|u|_{1} .
$$

$\rho_{0}, c_{s 0},|u|_{0}$ and $v_{0}$ are bulk properties of the BEC in the absence of a simulated gravitational wave, as above. It should be noted that these bulk properties are not necessarily constant in space or time; they are just the natural evolution of the BEC with no simulated gravitational wave disturbing them. Checking the normalization in Eq. (24), we see that

$$
v_{0}=\sqrt{c^{2}-\epsilon^{2}\left(v_{1}^{2}+v_{2}^{2}\right)}=c+\mathcal{O}\left(\epsilon^{2}\right)
$$

so, to first order, $v_{0}=c$. With this flow velocity, the acoustic metric is

$$
G_{\mu \nu}^{(\operatorname{sim})}=\frac{\rho c}{c_{s}}\left(\begin{array}{cccc}
-c_{s}^{2} / c^{2} & \epsilon r v_{1} / c & \epsilon r v_{2} / c & 0 \\
\epsilon r v_{1} / c & & & \\
\epsilon r v_{2} / c & & \mathbb{I}_{3} & \\
0 & &
\end{array}\right)
$$

to first order in $\epsilon$. Comparison of Eqs. (53) and (59) suggests that the velocity perturbation functions should take the form

$$
\begin{aligned}
& v_{1}=\frac{1}{2} \partial_{x} H_{00}, \\
& v_{2}=\frac{1}{2} \partial_{y} H_{00} .
\end{aligned}
$$


and the speed of sound perturbation must be

$$
c_{s 1}^{2}=c^{2}\left(1+r_{0}\right) h_{00} .
$$

As in Sec. III A, these conditions result in a conformal simulation, with the conformal factor

$$
G_{\mu \nu}^{(\mathrm{sim})}=\left(1+\epsilon \frac{\rho_{1}}{\rho_{0}}\right) G_{\mu \nu}^{(g w)} .
$$

If all components of both effective metrics are to match, there is no way to avoid this conformal factor. The form of the density perturbation $\rho_{1}$ required to implements Eqs. (60) and (61) will be calculated in Sec. III B 3. The conformal factor will be discussed further in Sec. III C.

\section{Bulk properties for simulation}

To implement the normalized velocity profile given above, we must calculate the restrictions placed on the other bulk properties of the condensate. From Eq. (25), we can derive the velocity normalization required for irrotational flow. We find that

$$
|u|(t, \boldsymbol{x})=|u|_{0}-\frac{\epsilon}{2 c} \partial_{t}\left(|u|_{0} H_{00}\right),
$$

where $\boldsymbol{x}$ represents all spatial dimensions $(x, y$ and $z)$. As in Sec. III A, in the limit of $\epsilon \rightarrow 0$, the BEC is stationary. Hence, the chemical potential is constant, so $|u|_{0}$ is constant in both space and time. Similarly, from these results and the continuity equation, Eq. (B16), we can derive the form of the density and its perturbation. We find that the density and its perturbation have the form

$$
\rho_{0}(t, \boldsymbol{x})=\frac{\alpha(\boldsymbol{x})}{|u|_{0}}
$$

and

$$
\begin{aligned}
\rho_{1}= & -\rho_{0}\left(\frac{|u|_{1}}{|u|_{0}}+\frac{c}{2}\left[\left(x h_{+}+y h_{\times}\right) \frac{\partial_{x} \rho_{0}}{\rho_{0}}\right.\right. \\
& \left.\left.+\left(x h_{\times}-y h_{+}\right) \frac{\partial_{y} \rho_{0}}{\rho_{0}}\right]\right),
\end{aligned}
$$

where $\alpha(\boldsymbol{x})$ is some arbitrary function of integration, encoding the spatial shape of the BEC cloud. It should be noted that the results of this section are not fundamental restrictions on the bulk properties on the BEC; rather, they are conditions that must be imposed in an experiment to facilitate the implementation of the desired flow velocities and speed of sound. As in Sec. III A, all of these conditions cannot be satisfied with an arbitrary interaction strength $\lambda$. Taking the same approach as above, we define a "perturbed" interaction strength

$$
\lambda=\lambda_{0}+\epsilon \lambda_{1} .
$$

In general from Eqs. (22) and (23), we must have

$$
\lambda_{1}=\lambda_{0}\left(2 \frac{|u|_{1}}{|u|_{0}}-\frac{\rho_{1}}{\rho_{0}}+\frac{c_{s 1}^{2}}{r_{0} c_{s 0}^{2}}\right) .
$$

With the results of this section, this expression becomes

$$
\begin{aligned}
\lambda_{1}= & \lambda_{0}\left[\left(\frac{c^{2}}{c_{s 0}^{2}}\left[1+\frac{1}{r_{0}}\right]-\frac{3}{2}\right) h_{00}\right. \\
& \left.-\frac{1}{2}\left(\left(x h_{+}+y h_{\times}\right) \frac{\partial_{x} \rho_{0}}{\rho_{0}}+\left(x h_{\times}-y h_{+}\right) \frac{\partial_{y} \rho_{0}}{\rho_{0}}\right)\right] .
\end{aligned}
$$

Using Eq. (27), this corresponds to an external potential

$$
\begin{aligned}
V= & V_{0}+\epsilon\left\{-\lambda_{0} \rho_{1}-\lambda_{1} \rho_{0}+\frac{2 m^{2}}{\hbar^{2}}|u|_{0}|u|_{1}\right. \\
& \left.+\frac{1}{\sqrt{\rho_{0}}}\left[\frac{\square \sqrt{\rho_{0}}}{\sqrt{\rho_{0}}}+\square\right] \frac{\rho_{1}}{2 \sqrt{\rho_{0}}}\right\} .
\end{aligned}
$$

\section{Static bulk solution}

Consider a BEC trapped in a uniform box potential with infinite potential walls. In such a case, the density of the BEC is approximately constant in space everywhere inside the box, apart from a region close to the boundaries of the trap, where the density goes to zero. The width of this boundary region is given by the healing length defined above in Eq. (15). However, as stated in the motivation for the definition of Eq. (15), we are interested in perturbations whose wavelength far exceeds the healing length. Hence, for the perturbations we are considering, in a uniform box potential, we can assume constant density everywhere. This is also assumed in the detector proposal [25]. As in Sec. III A, it seems most reasonable to require that $\rho_{0}$ be constant in time, and thus $|u|_{0}$ is also. In this case, the perturbed bulk properties required to simulate a gravitational wave derived above can be simplified somewhat. Applying these conditions, we find

$$
\begin{gathered}
|u|=|u|_{0}\left(1-\frac{\epsilon}{2} h_{00}\right), \\
\rho=\rho_{0}\left(1+\frac{\epsilon}{2} h_{00}\right),
\end{gathered}
$$

implemented with

$$
\begin{gathered}
\lambda_{1}=\lambda_{0}\left(\frac{c^{2}}{c_{s 0}^{2}}\left[1+\frac{1}{r_{0}}\right]-\frac{3}{2}\right) h_{00}, \\
V_{1}=-\left[\lambda_{0} \rho_{0}\left(\frac{c^{2}}{c_{s 0}^{2}}\left[\frac{3}{2}+\frac{1}{r_{0}}\right]-\frac{3}{2}\right)+\frac{1}{4 c^{2}} \partial_{t}^{2}\right] h_{00} .
\end{gathered}
$$


Using the definitions and conditions presented in Sec. III A 3, the nonrelativistic limit of these potential and interaction strength perturbations are

$$
\begin{gathered}
\lambda_{1}^{\mathrm{NR}}=-\frac{\lambda_{0}^{\mathrm{NR}}}{c_{s 0}^{2}}\left(2 x y \partial_{t}^{2} h_{\times}+\left(x^{2}-y^{2}\right) \partial_{t}^{2} h_{+}\right), \\
V_{1}^{\mathrm{NR}}=\frac{5 \lambda_{0}^{\mathrm{NR}} \rho_{0}}{2 c_{s 0}^{2}}\left(2 x y \partial_{t}^{2} h_{\times}+\left(x^{2}-y^{2}\right) \partial_{t}^{2} h_{+}\right) .
\end{gathered}
$$

\section{Conformal factor}

While the evolution of the phonon field is not generally conformally invariant in $3+1$ dimensions, there are conformally invariant properties that may be usefully measured and compared against theoretical predictions. The Weyl tensor is one of the standard examples of conformally invariant objects in the framework of general relativity, and for a GW spacetime has the form

$$
C_{\alpha \mu \beta \nu}=-k_{[\alpha} \bar{h}_{\mu][\nu} k_{\beta]},
$$

where $k_{\mu}$ is the wave vector of the $\mathrm{GW}$, and $\bar{h}_{\mu \nu}$ is the trace reversed perturbation defined as

$$
\bar{h}_{\mu \nu}=h_{\mu \nu}-\frac{1}{2} \eta_{\mu \nu} h_{\sigma}^{\sigma}
$$

for metric perturbation $h_{\mu \nu}$ and Minkowski metric $\eta_{\mu \nu}$ as defined above. This follows simply from the Riemann tensor for a GW spacetime [32],

$$
R_{\alpha \mu \beta \nu}=\frac{1}{2}\left(h_{\alpha \nu, \mu \beta}+h_{\mu \beta, \nu \alpha}-h_{\mu \nu, \alpha \beta}-h_{\alpha \beta, \mu \nu}\right) .
$$

In the TT gauge, the elements of the Weyl tensor have simple forms such as

$$
C_{0101}=-k_{z}^{2} h_{+}, \quad C_{0102}=-k_{z}^{2} h_{\times},
$$

which can be measured by the detector and compared against experimental parameters of the simulation.

\section{EXAMPLES OF GW SOURCES}

\section{A. Nonaxisymmetric neutron star}

Rotating neutron stars are one of the strongest predicted sources of continuous GWs [39]. Any imperfections in the symmetry of the mass distribution of a neutron star generate gravitational radiation as the star spins. The simplest case of a nonaxisymmetric neutron star spinning down has strain functions of the form [40]

$$
\epsilon h_{+}(t)=h_{0}\left(\frac{1+\cos ^{2} l}{2}\right) \cos \Phi(t)
$$

$$
\epsilon h_{\times}(t)=h_{0} \cos \imath \sin \Phi(t),
$$

where $l$ is the inclination of the neutron star's rotation axis to the line of sight, the phase evolution is

$$
\Phi(t)=\Phi_{0}+2 \pi f\left(t-t_{0}\right)
$$

for rotation frequency $f / 2$ and reference time $t_{0}$, and the amplitude $h_{0}$ is

$$
h_{0}=\frac{4 \pi^{2} G}{c^{4}} \frac{I_{z z} \epsilon_{x y} f^{2}}{d}
$$

with ellipticity

$$
\epsilon_{x y}=\frac{I_{x x}-I_{y y}}{I_{z z}}
$$

where $I_{i i}$ is the moment of inertia of the neutron star about some $i$ axis, $d$ is the distance to the neutron star and $G$ is Newton's gravitational constant. This coordinate system is defined such that the axis of rotation is parallel to the $z$ axis. On the timescale of a detection event, the frequency is constant to very good approximation, so terms in $\partial_{t} f$ in the phase are ignored [40]. The signal emitted by such a neutron star can be directly simulated with the interaction and external potential perturbations

$\lambda_{1}=\frac{\lambda_{0} f^{2} h_{0}}{2 r_{0} c^{2}}\left(2 x y \cos \iota \sin \Phi+\left(x^{2}-y^{2}\right)\left(\frac{1+\cos ^{2} \iota}{2}\right) \cos \Phi\right)$,

$$
V_{1}=-\lambda_{1} \rho
$$

\section{B. Compact binary coalescence}

The first direct experimental proof of the existence of GWs was recently reported by the LIGO Collaboration in [17], with the measurement of the GW signature of the final moments of a compact binary inspiral involving two black holes. These black holes were approximately 29 and 36 times the mass of the sun respectively, and 3 solar masses in energy were radiated in the form of GWs in the inspiral and collision. The form of the emitted gravitational radiation during the collision in the "strong gravity regime" must be calculated numerically, but the radiation emitted during the well-separated inspiral phase, and the ringdown after coalescence, has well-known solutions.

\section{Inspiral}

During the inspiral of a compact binary system, while the two compact objects are still well separated, the gravitational radiation far from the binary system has the form [32] 


$$
\begin{array}{r}
\epsilon h_{+}(t)=2\left(1+\cos ^{2} \imath\right) \frac{\mu}{d}[\pi M f(t)]^{2 / 3} \cos [2 \pi F(t)], \\
\epsilon h_{\times}(t)=4 \cos \imath \frac{\mu}{d}[\pi M f(t)]^{2 / 3} \sin [2 \pi F(t)],
\end{array}
$$

where $\iota$ is the inclination axis of inspiral axis to detector, $M=M_{1}+M_{2}$ and $\mu=M_{1} M_{2} / M^{2}$ for the two masses $M_{1}$ and $M_{2}, d$ is the distance from the inspiral barycenter to the detector,

$$
F(t)=\int^{t} f\left(t^{\prime}\right) d t^{\prime}
$$

and

$$
f(t)=\frac{1}{\pi}\left[\frac{5}{256} \frac{1}{\mu M^{2 / 3}} \frac{1}{\left(t_{0}-t\right)}\right]^{3 / 8}
$$

with some reference time $t_{0}$. This is a sinusoidal signal whose amplitude and frequency increase as the time $t$ reaches the reference time $t_{0}$, i.e., the time of collision. This is the characteristic "chirp" observed by the LIGO Collaboration in [17-19]. To directly simulate this metric, the corresponding interaction and external potential perturbations are

$$
\begin{aligned}
\lambda_{1}= & -\frac{\lambda_{0}}{r_{0} c^{2}} \frac{\mu}{d}(\pi M f)^{2 / 3}\left\{4 x y \cos \imath\left[\left(\frac{7 \pi f}{4\left(t_{0}-t\right)}\right) \cos (2 \pi F)+\left(\frac{5}{16\left(t_{0}-t\right)^{2}}-(2 \pi f)^{2}\right) \sin (2 \pi F)\right]\right. \\
& \left.+\left(x^{2}-y^{2}\right)\left(1+\cos ^{2} \imath\right)\left[\left(\frac{5}{16\left(t_{0}-t\right)^{2}}-(2 \pi f)^{2}\right) \cos (2 \pi F)-\left(\frac{7 \pi f}{4\left(t_{0}-t\right)}\right) \sin (2 \pi F)\right]\right\},
\end{aligned}
$$

$$
V_{1}=-\lambda_{1} \rho
$$

\section{Ringdown}

After a binary system with sufficient mass to form a black hole has collided and coalesced, the resulting black hole rotates due to conservation of angular momentum. The ringdown of the coalesced object into a stable rotating black hole can thus be modeled as a perturbed Kerr black hole. The simplest single-mode ringdown of a Kerr black hole has strain functions of the form [41]

$$
\begin{array}{r}
\epsilon h_{+}(t)=\frac{\mathcal{A}}{d}\left(1+\cos ^{2} \imath\right) e^{\left(\Phi_{0}-\Phi(t)\right) / 2 Q} \cos \Phi(t), \\
\epsilon h_{\times}(t)=\frac{\mathcal{A}}{d}(2 \cos \imath) e^{\left(\Phi_{0}-\Phi(t)\right) / 2 Q} \sin \Phi(t),
\end{array}
$$

where

$$
\Phi(t)=\Phi_{0}+2 \pi f\left(t-t_{0}\right)
$$

as above, $l$ is still the inclination angle of rotation axis to the detector, $d$ is the distance from the source to the detector, and $Q$ is the "quality factor" fitted numerically with

$$
Q=0.7000+1.4187(1-\hat{a})^{-0.4990}
$$

for spin parameter $\hat{a}=c S / G M^{2}$, with spin angular momentum $S$. The GW amplitude $\mathcal{A}$ is given by

$$
\mathcal{A}=\frac{G M}{c^{2}} \sqrt{\frac{5 \varepsilon}{2}} Q^{-1 / 2} F(Q)^{-1 / 2} g(\hat{a})^{-1 / 2}
$$

where $F(Q)=1+1 / 4 Q^{2}, g(\hat{a})=1.5251-1.1568(1-\hat{a})^{0.1292}$ and $\varepsilon$ is the fraction of the black hole mass radiated away.
Functionally, this is a decaying sinusoid of constant frequency. The corresponding interaction and external potential perturbations for simulation are

$$
\begin{gathered}
\lambda_{1}=-\frac{\lambda_{0}}{r_{0} c^{2}}\left\{2 x y \cos \imath\left(\left[\frac{1}{4 Q^{2}}-1\right] \sin \Phi-\frac{1}{Q} \cos \Phi\right)\right. \\
\left.+\frac{1}{2}\left(x^{2}-y^{2}\right)\left(1+\cos ^{2} \iota\right)\left[\frac{1}{Q} \sin \Phi+\left(\frac{1}{4 Q^{2}}-1\right) \cos \Phi\right]\right\} \\
\times \frac{\mathcal{A}}{d}(2 \pi f)^{2} e^{\left(\Phi_{0}-\Phi\right) / 2 Q}, \\
V_{1}=-\lambda_{1} \rho .
\end{gathered}
$$

\section{REDUCTION TO $1+1$}

In this section, we restrict ourselves to an effective 1-dimensional field to compare to earlier work in [16]. In an effective $1+1$-dimensional spacetime, the GW metric reduces to

$$
g_{\mu \nu}=\left(\begin{array}{cc}
-1-\epsilon x^{2} \partial_{t}^{2} h_{+} / 2 c^{2} & 0 \\
0 & 1
\end{array}\right)
$$

To simulate this, the speed of sound is chosen as

$$
c_{s}^{2}=c_{s 0}^{2}\left(1-\epsilon \frac{x^{2}}{2} \partial_{t}^{2} h_{+}\right)
$$

and a time scaling of

$$
c_{s 0} \tau=\left(\frac{c_{s 0}^{2}}{c^{2}}\right) c t
$$


results in a simulation

$$
G_{\mu^{\prime} \nu^{\prime}}^{(\mathrm{SIM})}=\frac{\rho c}{c_{s}}\left(\begin{array}{cc}
-1-\epsilon x^{2} \partial_{t}^{2} h_{+} / 2 c^{2} & 0 \\
0 & 1
\end{array}\right) .
$$

In $1+1$ dimensions, the equations of motion are conformally independent, so this is an exact simulation. Following the same procedure as in Sec. III A, we require that the flow velocity normalization be completely constant and conclude that the density is constant in time. The interaction strength and external potential perturbations required to implement this are then

$$
\begin{gathered}
\lambda_{1}=-\frac{\lambda_{0}}{r_{0} c^{2}}\left(\frac{x^{2}}{2} \partial_{t}^{2} h_{+}\right), \\
V_{1}=\frac{\lambda_{0} \rho}{r_{0} c^{2}}\left(\frac{x^{2}}{2} \partial_{t}^{2} h_{+}\right) .
\end{gathered}
$$

We must stress that this is an effective $1+1$-dimensional theory, and care must be taken when dealing with the actual field dynamics. Although this seems to work at the level of the metric, a naive suppression of the remaining spatial dimensions cannot be done due to the fact that the conformal factor is dimensionally dependent, and diverges when the number of spatial dimensions is exactly 1 [42]. Nevertheless, as long as the system is sufficiently constrained in the extra dimensions, e.g., in a highly elongated trap, a well-behaved effective $1+1$-dimensional system can always be constructed.

\section{CONCLUSION}

We have shown how to simulate a GW spacetime in $3+1$ dimensions for quantum excitations of a BEC, up to a conformal factor, as well as simulating the acoustic metric used in [25] to propose a GW detector. By making use of the "gauge freedom" of the GW metric corresponding to a linearized coordinate transformation, we chose a frame in which the metric perturbation could be simulated by perturbing the speed of sound in the BEC. We then examined the restrictions this places on other bulk properties through the continuity equation and experimental limitations, and calculated the external and interaction potential perturbations needed to implement such a simulation in the lab. Although the simulated metric is related to the target metric by a nonconstant conformal factor, we show that there are still useful properties that can be measured and tested in an experiment. We also give explicit expressions for the simulation of GWs from various sources. This work generalizes the results of [16] and presents a complementary approach to simulation in effectively $1+1$-dimensional BECs.

The results presented here can also be derived in the context of an explicitly nonrelativistic treatment of a BEC, such as that derived in [42]. In a nonrelativistic BEC, phonons on the BEC still propagate on a Lorentzian effective spacetime described by an acoustic metric, but this metric is necessarily spatially conformally flat. We consider a BEC in a covariant formalism in this paper to match the approach of [25-27] for the simulation in Sec. III B, and to express the interaction of a BEC with GWs in a natural way. As explained in Sec. II B, the simulation of GWs presented in this paper does not rely on the relativistic nature of the BEC or any relativistic effects, nor do the perturbations to the external and interaction potentials disappear in the nonrelativistic limit.

We have studied GWs in the context of perturbations around a flat spacetime metric and assuming GWs to be far outside the source. Other interesting simulations could involve GWs propagating on curved backgrounds, such as black holes $[9,10]$ or during inflation [43], or in strongfield regimes. Furthermore, since phonons are quantum quasiparticles, this opens up the possibility of studying predicted effects of quantum field theory in curved spacetime, such as how a GW may affect the entanglement of quantum systems, a phenomena that is utilized in the BEC GW detector proposed in [25]. This, therefore, also presents a potential, and fully configurable, testing environment for this $\mathrm{GW}$ detector metrological scheme. To obtain a full simulation of the GW detector, we need a better understanding of the effect of the GW on the bulk of the BEC. As mentioned in the conclusion of [16], an experimental simulation of the effect of a large amplitude $\mathrm{GW}$ and subsequent detection of phonons would also be a proof-of-concept demonstration of the generation of phonons by GWs as predicted in [25].

\section{ACKNOWLEDGMENTS}

The authors thank Joel Lindkvist and David Edward Bruschi for helpful discussion and comments. D. H. acknowledges funding from the Vienna Doctoral Program on Complex Quantum Systems (CoQuS) under the Austrian Science Fund (FWF) project code W 1210-N25. T. B. acknowledges funding from CONACYT under project code 261699/359033. D. R. thanks the Alexander von Humboldt Foundation for supporting his research with their Feodor Lynen Fellowship. R. H. and I. F. would like to acknowledge that this project was made possible through the support of the grant "Leaps in cosmology: gravitational wave detection with quantum systems" (Grant No. 58745) from the John Templeton Foundation. The opinions expressed in this publication are those of the authors and do not necessarily reflect the views of the John Templeton Foundation.

\section{APPENDIX A: SIMULATION IN ALTERNATIVE COORDINATES}

As explained in Sec. III B, it is possible to simulate the effect of a GW starting with no flows in Fermi normal coordinates. This solution is presented here. As above, let the density, speed of sound and flow velocity normalization be respectively described as 


$$
\begin{gathered}
\rho=\rho_{0}+\epsilon \rho_{1}, \\
c_{s}^{2}=c_{s 0}^{2}+\epsilon c_{s 1}^{2},
\end{gathered}
$$

and

$$
|u|=|u|_{0}+\epsilon|u|_{1},
$$

where $\epsilon$ is the small parameter defined above. $\rho_{0}, c_{s 0}$, and $|u|_{0}$ are bulk properties of the BEC in the absence of a simulated GW.

\section{Effective metric with background gravitational wave}

In Fermi normal coordinates, we consider the flows in the $\mathrm{BEC}$ to be $v_{\mu}=-v_{0} \delta_{\mu}^{0}$. With the normalization equation [Eq. (24)], we can determine the function $v_{0}$ as

$$
\begin{aligned}
g^{\mu \nu} v_{\mu} v_{\nu} & =-c^{2} \\
g^{\mu \nu} v_{\mu} v_{\nu} & =\left(\eta^{\mu \nu}-\epsilon h^{\mu \nu}\right) v_{0} \delta_{\mu}^{0} v_{0} \delta_{\nu}^{0} \\
& =\left(-1+\epsilon h_{00}\right) v_{0}^{2} \\
& =-c^{2} \\
\Rightarrow & v_{0}^{2}=c^{2}\left(1+\epsilon h_{00}\right) .
\end{aligned}
$$

Then, with a background GW, the acoustic metric has the form

$G_{\mu \nu}^{(g w)}=\frac{\rho_{0} c}{c_{s 0}}\left(\begin{array}{cc}-c_{s 0}^{2} / c^{2}\left(1+\epsilon h_{00}\right) & \mathbf{0}^{\mathrm{T}} \\ \mathbf{0} & \mathbb{I}_{3}\end{array}\right)+\mathcal{O}\left(\epsilon^{2}\right)$.

\section{Effective metric with simulated gravitational wave}

Consider the background metric $g_{\mu \nu}$ in Fermi normal coordinates to be the flat Minkowski metric $\eta_{\mu \nu}$ defined as in Eq. (1), with no background $\mathrm{GW}\left(h_{\mu \nu}=0\right)$. In these coordinates, consider the case where there are no flows on the BEC, so the flow velocity is

$$
\left.v\right|_{h \rightarrow 0}=(c, 0,0,0)
$$

To simulate the effect of a GW, we perturb the bulk properties of the BEC. The acoustic metric is

$$
G_{\mu \nu}^{(\operatorname{sim})}=\frac{\rho c}{c_{s}}\left(\begin{array}{cc}
-c_{s}^{2} / c^{2} & \mathbf{0}^{\mathrm{T}} \\
\mathbf{0} & \mathbb{I}_{3}
\end{array}\right)+\mathcal{O}\left(\epsilon^{2}\right) .
$$

\section{Simulation}

Comparison of Eqs. (A6) and (A8) suggests that, to simulate a background GW in these coordinates, the speed of sound should be modulated as

$$
c_{s}^{2}=c_{s 0}^{2}\left(1+\epsilon h_{00}\right) .
$$

As with the simulation presented in the main body of the paper, this simulation differs from an exact simulation by a conformal factor:

$$
G_{\mu \nu}^{(\operatorname{sim})}=\left(1+\epsilon\left[\frac{\rho_{1}}{\rho_{0}}-\frac{h_{00}}{2}\right]\right) G_{\mu \nu}^{(g w)} .
$$

\section{a. Bulk properties for simulation}

To implement the normalized velocity profile given above, we must calculate the restrictions placed on the other bulk properties of the condensate. From Eq. (25),

$$
|u|=|u|_{0}(t)
$$

and from Eq. (B16),

$$
\rho_{0}(t, \boldsymbol{x})=\frac{\alpha(\boldsymbol{x})}{|u|_{0}(t)}
$$

As in Sec. III A, it seems most reasonable to require that $\partial_{t} \rho_{0}=0$ and so $|u|_{0}$ is completely constant. Defining a "perturbed" interaction strength as

$$
\lambda=\lambda_{0}+\epsilon \lambda_{1}
$$

the results of this section and Eqs. (22) and (23) are simultaneously satisfied if

$$
\lambda_{1}=\frac{\lambda_{0}}{r_{0}} h_{00}
$$

This can be implemented together with the external potential

$$
V_{1}=-\frac{\lambda_{0} \rho}{r_{0}} h_{00}
$$

\section{APPENDIX B: ACOUSTIC METRIC WITH GENERAL BACKGROUND METRIC}

In $[13,34]$, the acoustic metric is derived for a flat Minkowski background metric. Since we require the same for a general background metric, in this Appendix we extend the acoustic metric to the case where the background metric is not necessarily flat.

\section{Equations of motion and basic approximations}

\section{a. Lagrangian}

The Lagrangian density for an interacting massive complex scalar field $\hat{\Phi}$ on a (in general curved) background with metric $g_{\mu \nu}$ may be written as 


$$
\begin{aligned}
\mathcal{L}= & -\sqrt{-g}\left\{g^{\mu \nu} \partial_{\mu} \hat{\Phi}^{\dagger} \partial_{\nu} \hat{\Phi}+\left(\frac{m^{2} c^{2}}{\hbar^{2}}+V\right) \hat{\Phi}^{\dagger} \hat{\Phi}\right. \\
& \left.+U\left(\hat{\Phi}^{\dagger} \hat{\Phi}, \lambda_{i}\right)\right\}
\end{aligned}
$$

where $m$ is the mass, the external potential $V$ is generally a function of space and time, and the interaction potential $U$ depends on coupling constants $\lambda_{i}$ which are also in principle functions of space and time. The background metric $g_{\mu \nu}$ cannot be completely general; we restrict ourselves to spacetimes with sufficiently weak curvature such that Bose-Einstein condensation can still be well defined. Further restrictions on the metric will be given in Appendix B $2 \mathrm{~b}$. The interaction potential $U$ can be expanded as

$U\left(\hat{\Phi}^{\dagger} \hat{\Phi}, \lambda_{i}\right)=\frac{1}{2 !} \lambda_{2} \hat{\Phi}^{\dagger} \hat{\Phi}^{\dagger} \hat{\Phi} \hat{\Phi}+\frac{1}{3 !} \lambda_{3} \hat{\Phi}^{\dagger} \hat{\Phi}^{\dagger} \hat{\Phi}^{\dagger} \hat{\Phi} \hat{\Phi} \hat{\Phi}+\cdots$

We will consider only the first term of $U$ corresponding to two-particle interactions, and ignore further terms corresponding to interactions with three or more particles. For notational convenience, we will drop the label on $\lambda_{2}$ so

$$
U\left(\hat{\Phi}^{\dagger} \hat{\Phi}, \lambda_{i}\right) \approx \frac{1}{2} \lambda \hat{\Phi}^{\dagger} \hat{\Phi}^{\dagger} \hat{\Phi} \hat{\Phi} .
$$

The Euler-Lagrange equation for $\hat{\Phi}^{\dagger}$ is

$$
\left[\square_{g}-\left(\frac{m^{2} c^{2}}{\hbar^{2}}+V\right)-\lambda \hat{\Phi}^{\dagger} \hat{\Phi}\right] \hat{\Phi}=0
$$

where

$$
\square_{g} \hat{\Phi}=\frac{1}{\sqrt{-g}} \partial_{\mu}\left(\sqrt{-g} g^{\mu \nu} \partial_{\nu} \hat{\Phi}\right)
$$

and $g$ is the determinant of $g_{\mu \nu}$.

\section{b. Approximations}

We now let this field $\hat{\Phi}$ represent a Bose-Einstein condensate and make the Bogoliubov approximation to separate the "condensed fraction" of the field $\phi$ from a small "uncondensed fraction" $\hat{\psi}$. This is done multiplicatively as

$$
\hat{\Phi}=\phi(1+\hat{\psi})
$$

to simplify the equation for $\hat{\psi}$ later. As part of the Bogoliubov approximation, we say that

$$
\langle\hat{\Phi}\rangle=\phi \Rightarrow\langle\hat{\psi}\rangle=0
$$

where $\langle\cdot\rangle$ is a nonequilibrium average. Taking the average of Eq. (B4),

$$
\begin{aligned}
& {\left[\square_{g}-\left(\frac{m^{2} c^{2}}{\hbar^{2}}+V\right)-\lambda|\phi|^{2}\right] \phi-\lambda|\phi|^{2} \phi\left[\langle\hat{\psi} \hat{\psi}\rangle+2\left\langle\hat{\psi}^{\dagger} \hat{\psi}\right\rangle\right.} \\
& \left.\quad+\left\langle\hat{\psi}^{\dagger} \hat{\psi} \hat{\psi}\right\rangle\right]=0 .
\end{aligned}
$$

We now take the Popov approximation

$$
\langle\hat{\psi} \hat{\psi}\rangle=0=\langle\hat{\psi} \hat{\psi} \hat{\psi}\rangle
$$

and require that the density of excited atoms be much smaller than the density of mean-field atoms, i.e.,

$$
\left\langle\hat{\psi}^{\dagger} \hat{\psi}\right\rangle \ll 1 .
$$

This results in a nonlinear Klein-Gordon-like equation for the mean field $\phi$ :

$$
\left[\square_{g}-\left(\frac{m^{2} c^{2}}{\hbar^{2}}+V\right)-\lambda|\phi|^{2}\right] \phi=0 .
$$

This is a curved spacetime generalization of the GrossPitaevskii equation. In flat spacetime [where the metric is the Minkowski metric $\eta_{\mu \nu}$ defined in Eq. (1)] and in the nonrelativistic limit, we can replace $\phi$ with a lower energy field

$$
\phi=\varphi e^{i m c^{2} t / \hbar}
$$

and take the limit of $c \rightarrow \infty$. Assuming that the energy of excitations in $\varphi$ is sufficiently low such that we can ignore terms of order $\partial_{t}^{2} \varphi$, the remaining terms of Eq. (B11) have the form

$$
i \hbar \partial_{t} \varphi=\left[-\frac{\hbar^{2}}{2 m} \nabla^{2}+V_{\mathrm{NR}}+g_{\mathrm{NR}}|\varphi|^{2}\right] \varphi
$$

where

$$
g_{\mathrm{NR}}=\frac{\hbar^{2}}{2 m} \lambda, \quad V_{\mathrm{NR}}=\frac{\hbar^{2}}{2 m} V,
$$

which is the usual time-dependent Gross-Pitaevskii equation.

\section{c. Continuity and velocity normalization equations}

If the mean field $\phi$ is written in the Madelung representation $\phi=\sqrt{\rho} e^{i \theta}$ and defining a flow velocity

$$
u^{\mu}=\frac{\hbar}{m} g^{\mu \nu} \partial_{\nu} \theta,
$$

then separating the real and imaginary components of Eq. (B11) results in two equations:

$$
\nabla_{\mu}\left(\rho u^{\mu}\right)=0,
$$




$$
-g_{\mu \nu} u^{\mu} u^{\nu}=c^{2}+\frac{\hbar^{2}}{m^{2}}\left\{V+\lambda \rho-\frac{\square_{g} \sqrt{\rho}}{\sqrt{\rho}}\right\} .
$$

Equation (B16) is a continuity equation, and can also be derived from the global phase $U(1)$ symmetry of the Lagrangian density in Eq. (B1). Equation (B17) allows us to directly relate the external and interaction potentials with the mean-field properties of the BEC without necessarily solving the full dynamics with Eq. (B11).

\section{Phonon equations}

\section{a. Equations for $\hat{\boldsymbol{\psi}}$}

Combining Eqs. (B4), (B6) and (B11), we find

$$
\left[i \hbar u^{\mu} \partial_{\mu}-\hat{T}_{\rho}-m c_{0}^{2}\right] \hat{\psi}=m c_{0}^{2} \hat{\psi}^{\dagger}
$$

where

$$
c_{0}^{2}=\frac{\hbar^{2}}{2 m^{2}} \lambda \rho
$$

and

$$
\hat{T}_{\rho} \hat{\psi}=-\frac{\hbar^{2}}{2 m \rho \sqrt{-g}} \partial_{\mu}\left(\rho \sqrt{-g} g^{\mu \nu} \partial_{\nu} \hat{\psi}\right)
$$

is a generalized kinetic operator, which reduces to the standard kinetic energy operator $T=-\left(\hbar^{2} / 2 m\right) \nabla^{2}$ for constant $\rho$ in the nonrelativistic flat spacetime limit. Note that we require the solution to Eq. (B11) to solve Eq. (B18) but not vice versa, as we are neglecting the backreaction of $\hat{\psi}$ on $\phi$. Taking the equivalent equation to Eq. (B18) for $\hat{\psi}^{\dagger}$ and combining these to eliminate $\hat{\psi}^{\dagger}$, we find

$$
\left(\left[i \hbar u^{\mu} \partial_{\mu}+\hat{T}_{\rho}\right] \frac{1}{c_{0}^{2}}\left[-i \hbar u^{\mu} \partial_{\mu}+\hat{T}_{\rho}\right]+2 m \hat{T}_{\rho}\right) \hat{\psi}=0 .
$$

It is important to note that although Eq. (B18) implies Eq. (B21), the converse is not true.

\section{b. Relative term strength}

The phonon equation Eq. (B21) can be expanded into four terms as

$$
\hat{T}_{1}+\hat{T}_{2}+\hat{T}_{3}+\hat{T}_{4}=0
$$

where

$$
\begin{gathered}
\hat{T}_{1}=i \hbar u^{\mu} \partial_{\mu} \frac{1}{c_{0}^{2}}\left[-i \hbar u^{\nu} \partial_{\nu}\right] \hat{\psi} \\
\hat{T}_{2}=i \hbar\left[u^{\mu} \partial_{\mu} \frac{1}{c_{0}^{2}} \hat{T}_{\rho}-\hat{T}_{\rho} \frac{1}{c_{0}^{2}} u^{\mu} \partial_{\mu}\right] \hat{\psi},
\end{gathered}
$$

$$
\hat{T}_{3}=\hat{T}_{\rho} \frac{1}{c_{0}^{2}} \hat{T}_{\rho} \hat{\psi}
$$

and

$$
\hat{T}_{4}=2 m \hat{T}_{\rho} \hat{\psi} .
$$

We make an eikonal approximation, where

$$
\left|\frac{\partial_{t} \rho}{\rho}\right| \ll \omega, \quad\left|\frac{\partial_{t} c_{0}}{c_{0}}\right| \ll \omega, \quad\left|\frac{\partial_{t} u^{\mu}}{u^{\mu}}\right| \ll \omega
$$

and the corresponding relations for variations in space as in the flat space case, but also

$$
\left|\frac{\partial_{t} g_{\mu \nu}}{g_{\mu \nu}}\right| \ll \omega, \quad\left|\frac{\partial_{t} g}{2 g}\right| \ll \omega
$$

with the corresponding relations for variations in space. Note that Eq. (B28) restricts the curvature of the metric with respect to the phonon mode frequencies. For linearized gravity and realistic phonon frequencies, this will always hold. Additionally, following $[13,35]$ we consider small momenta in the phononic regime, such that the dispersion relation is linear and terms quartic in $k$ can be neglected. With these approximations, $\hat{T}_{2}$ and $\hat{T}_{3}$ are negligible in comparison to $\hat{T}_{1}$ and $\hat{T}_{4}$, so we are left with

$$
\left[u^{\mu} \partial_{\mu} \frac{1}{c_{0}^{2}} u^{\nu} \partial_{\nu}+\frac{2 m}{\hbar^{2}} \hat{T}_{\rho}\right] \hat{\psi}=0
$$

Expanding Eq. (B29), we find an equation of the form

$$
\partial_{\mu}\left(f^{\mu \nu} \partial_{\nu} \hat{\psi}\right)=0
$$

where

$$
f^{\mu \nu}=\rho \sqrt{-g}\left[g^{\mu \nu}-\frac{u^{\mu} u^{\nu}}{c_{0}^{2}}\right]
$$

\section{c. Acoustic metric}

Equation (B30) has a form similar to a Klein-Gordon equation for a massless noninteracting scalar field $\hat{\psi}$ in a spacetime with an effective metric given by

$$
f^{\mu \nu}=\sqrt{-G} G^{\mu \nu} .
$$

Taking the determinant of this equation, we have

$$
G=-(-f)^{\frac{2}{n-2}}
$$

where $n$ is the total number of dimensions, noting that $f$ is the determinant of $f^{\mu \nu}$, but $G$ is the determinant of $G_{\mu \nu}$. Taking the determinant of $f^{\mu \nu}$, we find that

$$
\sqrt{-G}=\sqrt{-g} \rho^{\frac{n}{n-2}}\left(\frac{c}{c_{s}}\right)^{\frac{2}{n-2}}
$$


where the scalar speed of sound $c_{s}$ is defined as

$$
c_{s}^{2}=\frac{c^{2} c_{0}^{2}}{|u|^{2}+c_{0}^{2}} \text {. }
$$

Hence, defining normalized flow velocity as

$$
v^{\mu}=\frac{c}{|u|} u^{\mu},
$$

the inverse general acoustic metric is

$$
G^{\mu \nu}=\left(\frac{\rho c}{c_{s}}\right)^{-\frac{2}{n-2}}\left[g^{\mu \nu}+\left(1-\frac{c^{2}}{c_{s}^{2}}\right) \frac{v^{\mu} v^{\nu}}{c^{2}}\right]
$$

which can be inverted to define the general acoustic metric

$$
G_{\mu \nu}=\left(\frac{\rho c}{c_{s}}\right)^{\frac{2}{n-2}}\left[g_{\mu \nu}+\left(1-\frac{c_{s}^{2}}{c^{2}}\right) \frac{v_{\mu} v_{\nu}}{c^{2}}\right]
$$

[1] W. G. Unruh, Phys. Rev. Lett. 46, 1351 (1981).

[2] O. Lahav, A. Itah, A. Blumkin, C. Gordon, S. Rinott, A. Zayats, and J. Steinhauer, Phys. Rev. Lett. 105, 240401 (2010).

[3] C.-L. Hung, V. Gurarie, and C. Chin, Science 341, 1213 (2013).

[4] J. Steinhauer, Nat. Phys. 10, 864 (2014).

[5] S. Weinfurtner, E. W. Tedford, M. C. J. Penrice, W. G. Unruh, and G. A. Lawrence, Phys. Rev. Lett. 106, 021302 (2011).

[6] L.-P. Euvé, F. Michel, R. Parentani, and G. Rousseaux, Phys. Rev. D 91, 024020 (2015).

[7] T. G. Philbin, C. Kuklewicz, S. Robertson, S. Hill, F. König, and U. Leonhardt, Science 319, 1367 (2008).

[8] J. Steinhauer, Nat. Phys. 12, 959 (2016).

[9] B. Cropp, S. Liberati, and R. Turcati, Phys. Rev. D 94, 063003 (2016).

[10] R. Dey, S. Liberati, and R. Turcati, Phys. Rev. D 94, 104068 (2016).

[11] L. Giacomelli and S. Liberati, Phys. Rev. D 96, 064014 (2017).

[12] C. Barceló, S. Liberati, and M. Visser, Int. J. Mod. Phys. D 12, 1641 (2003).

[13] S. Fagnocchi, S. Finazzi, S. Liberati, M. Kormos, and A. Trombettoni, New J. Phys. 12, 095012 (2010).

[14] U. R. Fischer and R. Schützhold, Phys. Rev. A 70, 063615 (2004).

[15] S.-Y. Chä and U. R. Fischer, Phys. Rev. Lett. 118, 130404 (2017).

[16] T. Bravo, C. Sabín, and I. Fuentes, EPJ Quantum Techno. 2, 3 (2015).

[17] B. P. Abbott, R. Abbott, T. D. Abbott, M. R. Abernathy, F. Acernese, K. Ackley, C. Adams, T. Adams, P. Addesso, R. X. Adhikari, V. B. Adya, C. Affeldt, M. Agathos, K. Agatsuma, N. Aggarwal et al. (LIGO Scientific Collaboration and Virgo Collaboration), Phys. Rev. Lett. 116, 061102 (2016).

[18] B. P. Abbott, R. Abbott, T. D. Abbott, M. R. Abernathy, F. Acernese, K. Ackley, C. Adams, T. Adams, P. Addesso, R. X. Adhikari, V. B. Adya, C. Affeldt, M. Agathos, K. Agatsuma, N. Aggarwal et al. (LIGO Scientific Collaboration and Virgo Collaboration), Phys. Rev. Lett. 116, 241103 (2016).

[19] B. P. Abbott, R. Abbott, T. D. Abbott, F. Acernese, K. Ackley, C. Adams, T. Adams, P. Addesso, R. X. Adhikari, V. B. Adya, C. Affeldt, M. Afrough, B. Agarwal, M. Agathos, K. Agatsuma et al. (LIGO Scientific and Virgo Collaboration), Phys. Rev. Lett. 118, 221101 (2017).

[20] B. P. Abbott, R. Abbott, T. D. Abbott, F. Acernese, K. Ackley, C. Adams, T. Adams, P. Addesso, R. X. Adhikari, V. B. Adya, C. Affeldt, M. Afrough, B. Agarwal, M. Agathos, K. Agatsuma et al. (LIGO Scientific Collaboration and Virgo Collaboration), Phys. Rev. Lett. 119, 141101 (2017).

[21] B. P. Abbott, R. Abbott, T. D. Abbott, F. Acernese, K. Ackley, C. Adams, T. Adams, P. Addesso, R. X. Adhikari, V. B. Adya, C. Affeldt, M. Afrough, B. Agarwal, M. Agathos, K. Agatsuma et al. (LIGO Scientific Collaboration and Virgo Collaboration), Phys. Rev. Lett. 119, 161101 (2017).

[22] P. Jones, P. McDougall, and D. Singleton, Phys. Rev. D 95, 065010 (2017).

[23] M.-T. Jaekel, B. Lamine, A. Lambrecht, S. Reynaud, and P. M. Neto, in Proceedings Leiden 2006 (World Scientific, Leiden, Netherlands, 2006), p. 125.

[24] I. Fernandez-Corbaton, M. Cirio, A. Bse, L. Lamata, E. Solano, and G. Molina-Terriza, Sci. Rep. 5, 11538 (2015).

[25] C. Sabín, D. E. Bruschi, M. Ahmadi, and I. Fuentes, New J. Phys. 16, 085003 (2014).

[26] C. Sabín, J. Kohlrus, D. E. Bruschi, and I. Fuentes, EPJ Quantum Techno. 3, 8 (2016).

[27] R. Howl, L. Hackermüller, D. E. Bruschi, and I. Fuentes, arXiv:1607.06666v2.

[28] G. T. Moore, J. Math. Phys. (N.Y.) 11, 2679 (1970).

[29] S. A. Fulling and P. C. W. Davies, Proc. R. Soc. A 348, 393 (1976).

[30] R. Howl, C. Sabín, L. Hackermüller, and I. Fuentes, J. Phys. B 51, 015303 (2018).

[31] S. L. Braunstein and C. M. Caves, Phys. Rev. Lett. 72, 3439 (1994).

[32] C. W. Misner, K. S. Thorne, and J. A. Wheeler, Gravitation (W. H. Freeman and Company, San Francisco, 1973). 
[33] M. Maggiore, Gravitational Waves (Oxford University Press, Oxford, 2007).

[34] M. Visser and C. Molina-Paris, New J. Phys. 12, 095014 (2010).

[35] D. E. Bruschi, C. Sabín, A. White, V. Baccetti, D. K. L. Oi, and I. Fuentes, New J. Phys. 16, 053041 (2014).

[36] U. Schneider, L. Hackermüller, J. P. Ronzheimer, S. Will, S. Braun, T. Best, I. Bloch, E. Demler, S. Mandt, D. Rasch, and A. Rosch, Nat. Phys. 8, 213 (2012).

[37] U. Leonhardt, Nature (London) 415, 406 (2002).

[38] M. G. Richarte, I. G. Salako, J. P. M. Graça, H. Moradpour, and A. Övgün, Phys. Rev. D 96, 084022 (2017).

[39] J. Aasi, B. P. Abbott, R. Abbott, T. Abbott, M. R. Abernathy, T. Accadia, F. Acernese, K. Ackley, C. Adams, T. Adams,
P. Addesso, R. X. Adhikari, C. Affeldt, M. Agathos, N. Aggarwal et al. (LIGO Scientific Collaboration and Virgo Collaboration), Phys. Rev. D 90, 062010 (2014).

[40] C. Palomba, arXiv:1201.3176.

[41] J. Aasi, B. P. Abbott, R. Abbott, T. Abbott, M. R. Abernathy, F. Acernese, K. Ackley, C. Adams, T. Adams, P. Addesso, R. X. Adhikari, C. Affeldt, M. Agathos, N. Aggarwal, O. D. Aguiar et al. (LIGO Scientific Collaboration and Virgo Collaboration), Phys. Rev. D 89, 102006 (2014).

[42] C. Barceló, S. Liberati, and M. Visser, Living Rev. Relativity 8, 12 (2005).

[43] P. O. Fedichev and U. R. Fischer, Phys. Rev. Lett. 91, 240407 (2003). 